\title{
Hard prey, soft jaws and the ontogeny of feeding mechanics in the spotted ratfish Hydrolagus colliei
}

\author{
Daniel R. Huber ${ }^{1, *}$, Mason N. Dean ${ }^{2}$ and Adam P. Summers ${ }^{2}$ \\ ${ }^{1}$ Department of Biology, University of Tampa, 401 W. Kennedy Boulevard, \\ Box U, Tampa, FL 33606, USA \\ ${ }^{2}$ Ecology and Evolutionary Biology, 321 Steinhaus Hall, University of California, \\ Irvine, Irvine, CA 92697, USA
}

\begin{abstract}
The spotted ratfish Hydrolagus colliei is a holocephalan fish that consumes hard prey (durophagy) but lacks many morphological characters associated with durophagy in other cartilaginous fishes. We investigated its feeding biomechanics and biting performance to determine whether it can generate bite forces comparable with other durophagous elasmobranchs, how biting performance changes over ontogeny (21-44 cm SL) and whether biomechanical modelling can accurately predict feeding performance in holocephalans. Hydrolagus colliei can generate absolute and mass-specific bite forces comparable with other durophagous elasmobranchs (anterior $=104 \mathrm{~N}$, posterior $=191 \mathrm{~N}$ ) and has the highest jaw leverage of any cartilaginous fish studied. Modelling indicated that cranial geometry stabilizes the jaw joint by equitably distributing forces throughout the feeding mechanism and that positive allometry of bite force is due to hyperallometric mechanical advantage. However, bite forces measured through tetanic stimulation of the adductor musculature increased isometrically. The jaw adductors of $H$. collie $i$ fatigued more rapidly than those of the piscivorous spiny dogfish Squalus acanthias as well. The feeding mechanism of $H$. colliei is a volume-constrained system in which negative allometry of cranial dimensions leaves relatively less room for musculature. Jaw adductor force, however, is maintained through ontogenetic changes in muscle architecture.
\end{abstract}

Keywords: chimaera; feeding biomechanics; bite force; durophagy; scaling; ontogeny

\section{INTRODUCTION}

The robust, hypermineralized tooth plates of holocephalan fishes (Chondrichthyes: Holocephali) have branded them as hard prey specialists (Didier 1995; Grogan \& Lund 2004), although there is no experimental and relatively little dietary evidence to support this assertion (Johnson \& Horton 1972; Ebert 2003). Their tooth plates are arranged in three pairs: two on the upper jaws and one pair on the mandible, presenting two functionally separate regions of occlusion. The anterior parts of all the three pairs are 'nipping' blades, some with hypermineralized rods reinforcing the cutting edges. In contrast, the upper posterior plates and posterior portion of the mandibular plates form a broad, molariform-crushing surface. Both functional partitions of this dental battery show wear patterns consistent with processing hard prey in a variety of holocephalans (Didier 1995).

*Author for correspondence (dhuber@ut.edu).

This work is dedicated to the memory of G. Rau for inspiring a spirit of discovery and nurturing all fascinations, no matter how bizarre.
The several independent evolutions of a durophagous diet in the sister group to the holocephalans, the elasmobranchs (Chondrichthyes: Elasmobranchii), reveal a suite of morphological characters associated with consuming hard prey, including molariform teeth, hypertrophied jaw adductors and robust, wellcalcified jaws with high mechanical advantage (MA; Summers 2000; Summers et al. 2004; Huber et al. 2005; figure 1). The presence of a molariform or pavementlike dentition is strongly correlated with hard prey consumption (durophagy) in a number of elasmobranchs (sharks, skates and rays; Chondrichthyes: Elasmobranchii) such as the horn shark Heterodontus francisci and cownose ray Rhinoptera bonasus, which routinely consume prey spanning a wide range of molluscs, echinoderms and crustaceans (Segura-Zarzosa et al. 1997; Peterson et al. 2001). Although the posterior occlusal surface of their dentition is suited for crushing, holocephalans may not fit the elasmobranch model for durophagy - they have very poorly mineralized jaws and their jaw closing muscles are not obviously hypertrophied (Didier 1995).

If we assume holocephalans eat hard prey, the performance of their feeding mechanisms must be 


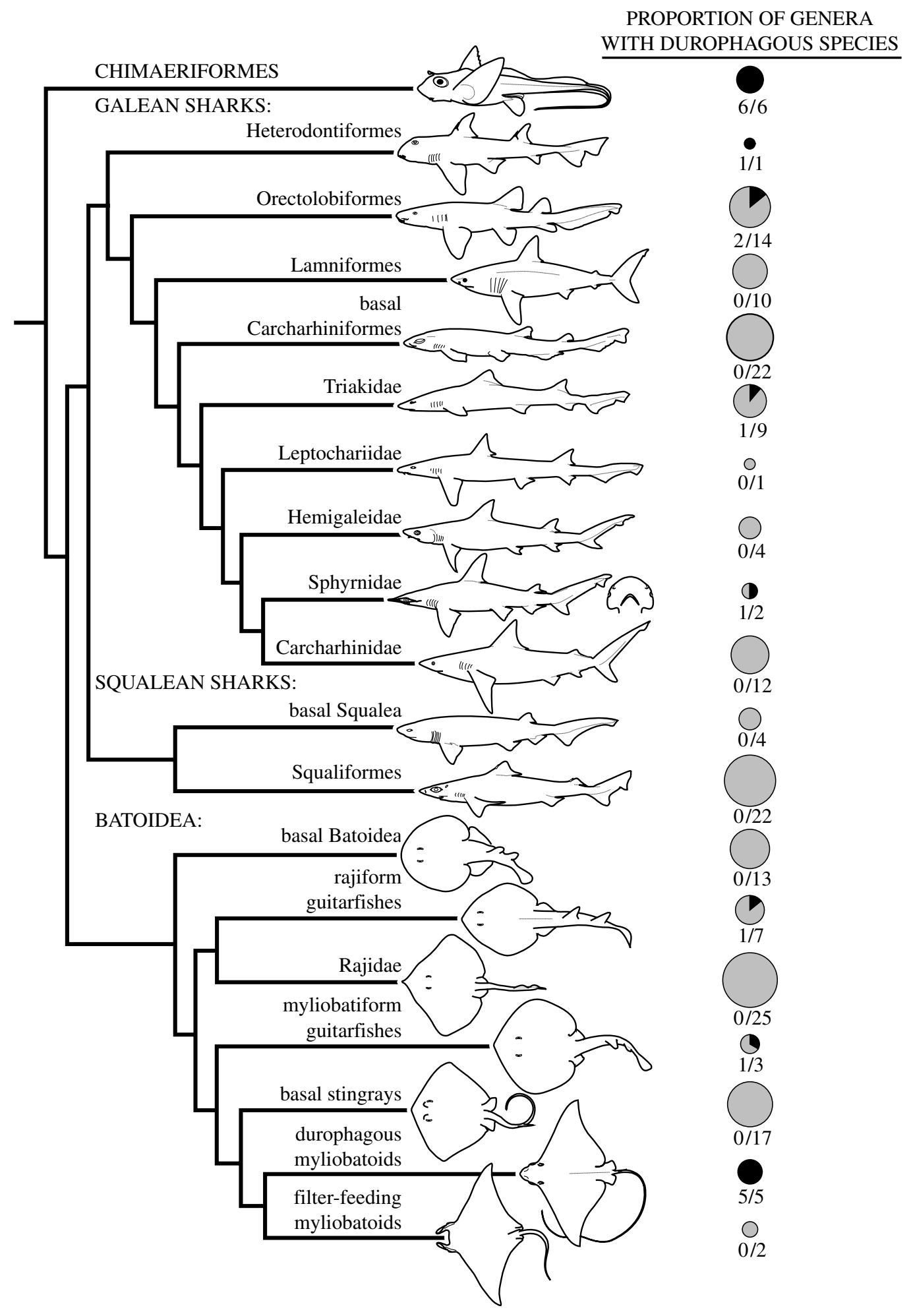

Figure 1. Cladogram of the cartilaginous fishes indicating the occurrence of hard prey consumption (durophagy). Circles to the right indicate the proportion of genera within a given taxonomic grouping which have durophagous species (black shade, durophagous; grey shade, non-durophagous; Compagno 2005; Dean et al. 2007b). Circle size is scaled to the total number of genera in a given taxonomic grouping. The topology of the cladogram is based on Compagno (2005), with some clades collapsed for simplicity.

comparable with that of the durophagous elasmobranchs. Performance (maximum bite force) associated with crushing hard prey has been accurately estimated using biomechanical modelling and muscle stimulation experiments in the horn shark $H$. francisci. These experiments clearly illustrate the causal relationship between morphology and function, providing an accurate way to estimate feeding performance that relies on cranial anatomy rather than observations of live animals in the field (Huber et al. 2005). Useful proxies for natural performance are particularly valuable for rare, intractable animals like holocephalans, which are mostly from abyssal depths and are difficult to keep alive under experimental conditions.

Given the practical difficulties associated with studying holocephalans and their incompatibility with the 
elasmobranch feeding model for durophagy, proxies for natural performance are essential to studying feeding in these enigmatic fishes. The spotted ratfish Hydrolagus colliei is an excellent representative holocephalan for investigating the links between morphology, performance and ecology, because it is found at relatively shallow depths (below $1000 \mathrm{~m}$ ) and can be maintained under experimental conditions for short periods of time. In addition, dietary data specifically from $H$. colliei show that they eat predominantly hard prey, including a large percentage of gastropod molluscs (Johnson \& Horton 1972; Wingert et al. 1979; Quinn et al. 1980). We expect that this species will be able to exert high bite forces, though perhaps at the cost of rapid fatigue to the jaw-adducting muscles as muscles specialized for force production often sacrifice stamina (Rome 1994). Investigation of the fatiguability of the jaw adductors may therefore clarify a characteristic relationship between performance and diet.

The goals of our study of the holocephalan feeding apparatus are to (i) estimate biting performance via biomechanical modelling of jaw leverage and muscle force generation and compare these hypotheses with bite force measurements recorded directly in muscle stimulation experiments, (ii) place this performance in the context of the life history of $H$. collie $i$ by measuring changes in bite force capability across a wide size range from juveniles to mature animals, (iii) measure the decrease in bite force production with repeated muscle stimulation as a proxy for whole muscle fatigue, and (iv) compare the biting performance of $H$. colliei with durophagous and non-durophagous elasmobranchs and bony fishes.

\section{MATERIAL AND METHODS}

\subsection{Experimental animals}

Eight H. colliei (21-44 cm SL) were collected by deepwater trawl in the San Juan Islands, WA, and housed at the University of Washington Friday Harbor Laboratories in a $2200 \mathrm{l}$ tank with a flow-through seawater system. Individuals were maintained on a diet of spot prawns Pandalus platyceros and sand lance Ammodytes hexapterus during the collection of tetanically stimulated bite force measurements (see below), after which they were euthanatized by an overdose of tricaine methanesulphonate (MS-222). Three spiny dogfish Squalus acanthias (48-62 cm SL) were caught and maintained in the same manner to compare the fatigue resistance of their jaw musculature with that of H. colliei. All husbandry and experimentation were performed in accordance with the guidelines of the Institutional Animal Care and Use Committee of the University of Washington.

\subsection{Theoretical bite force}

Bite force was estimated in $H$. colliei by modelling the forces generated by the adductor mandibulae anterior and posterior muscles and the lever mechanics of its feeding mechanism. The adductor mandibulae anterior, which comprises the bulk of the adductive musculature, is a white-fibred bipinnate muscle with two divisions that originate on the broad ethmoidal region of the cranium anterior to the orbit (Didier 1995; Dean et al. $2007 a$ ). The adductor mandibulae posterior is a much smaller parallel-fibred, red muscle located near the corner of the jaw (figure 2a). The jaw joint itself has been described as 'interlocking'; that is, the lower jaw has a double articulation with the quadrate process of the neurocranium (Didier 1995). A three-dimensional reconstruction from computed tomography scans of the head of $H$. colliei is available at the Digimorph website (www.digimorph.org).

Calculation of theoretical bite force requires an understanding of the cranial and muscle geometry. Prior to dissection, head width and height were measured as the distances between the lateral margins of the head and the distance from the dorsal to ventral margins of the head at the anterior margin of the eyes, respectively. Using the tip of the snout as the origin of a three-dimensional coordinate system, the origins and insertions of both muscles, as well as the positions of the jaw joint, and bite points at the anterior and posterior margins of the mandibular tooth plates were determined by measuring the distances of these points from the respective $X, Y$ and $Z$ planes intersecting the tip of the snout. Both the jaw-adducting muscles were then unilaterally excised. The adductor mandibulae posterior was bisected perpendicular to its centre of mass and digitally photographed in cross section, from which anatomical cross-sectional area $\left(A_{\mathrm{CSA}}\right)$ was measured using SigmaScan Pro v. 4.01 (SYSTAT Software, Inc., Point Richmond, CA, USA). The anatomical cross-sectional area was then multiplied by the specific tension of elasmobranch red muscle $\left(T_{\mathrm{SPR}}=142 \mathrm{kN} \mathrm{m}^{-2}\right.$; Lou et al. 2002$)$ to determine the theoretical maximum tetanic force $\left(P_{0}\right)$ produced,

$$
P_{0}=A_{\mathrm{CSA}} \times T_{\mathrm{SPR}} \text {. }
$$

The specific tension used is the available value most probably applicable to holocephalans, and specific tensions have been shown to be conservative in vertebrates. The bipinnate fibre architecture of the adductor mandibulae anterior made it necessary to determine this muscle's physiological cross-sectional area $\left(P_{\mathrm{CSA}}\right)$ in order to estimate its maximum tetanic force. $P_{\mathrm{CSA}}$ accounts for the fact that a portion of the force generated by muscle fibres that are not parallel to the central line of action of the muscle will be lost during contraction. In a bipinnate muscle, the force components normal to the muscle's line of action cancel out and do not contribute force to the action being performed. $P_{\mathrm{CSA}}$ estimates the portion of the muscle making a mechanically relevant input to a musculoskeletal system and is calculated using the equation

$$
P_{\mathrm{CSA}}=\frac{\text { muscle mass }}{\text { muscle density }} \times \cos \Theta \times \frac{1}{\text { fibre length }},
$$

where $\Theta$ is the average angle of pinnation from the central tendon of the muscle and the density of the fish muscle is $1.05 \mathrm{~g} \mathrm{~cm}^{-3}$ (Powell et al. 1984; Wainwright 1988). Preliminary analysis revealed considerably different fibre angles on either side of the central tendon of the adductor mandibulae anterior (figure $2 a$ ). 


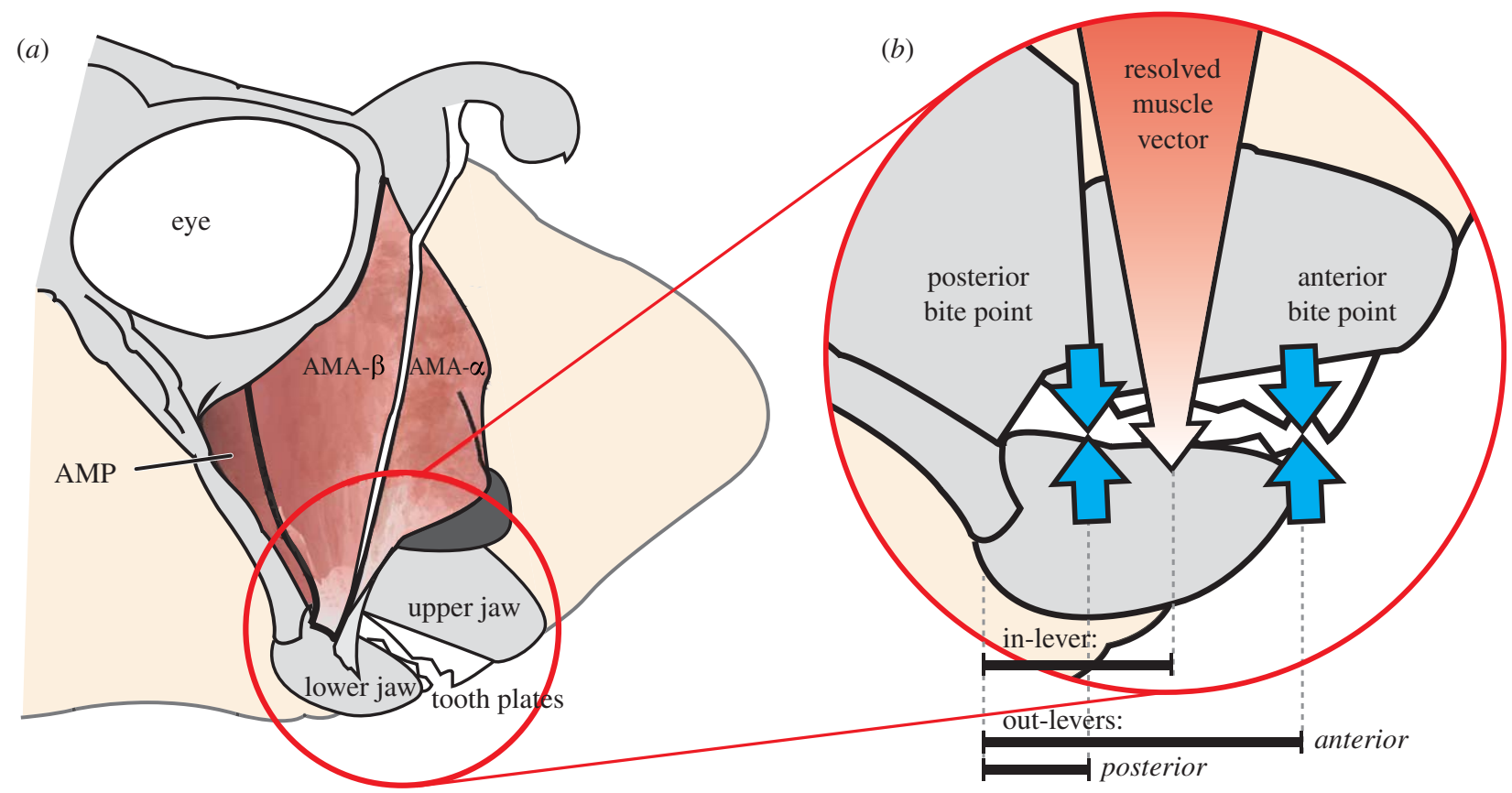

Figure 2. (a) Right lateral view of the cranial anatomy of $H$. colliei indicating the jaw structures and muscles used in the biomechanical modelling of the feeding mechanism. (b) Right lateral view of the jaws of $H$. colliei indicating the position of the resolved adductive muscle force vector and the anterior and posterior bite points. The distance between the jaw joint and the resolved adductive muscle force vector is the resolved in-lever for jaw adduction. The distances between the jaw joint and the anterior and posterior bite points are the out-levers for anterior and posterior biting, respectively. AMA- $\alpha$, adductor mandibulae anterior- $\alpha$; AMA- $\beta$, adductor mandibulae anterior- $\beta$; AMP, adductor mandibulae posterior.

Therefore, the $\alpha$ (anterior) and $\beta$ (posterior) subdivisions were investigated separately and $P_{\mathrm{CSA}}$ was determined for each. We digested the adductor mandibulae anterior$\alpha$ and $-\beta$ subdivisions in sulphuric acid and measured from digital images the lengths of seven fibres from each division of each individual using SigmaScan Pro v. 4.01. The means of these measurements were used in the calculation of physiological cross-sectional area for each individual. Theoretical maximum tetanic force of each division was determined as described above using the specific tension of elasmobranch white muscle $\left(T_{\mathrm{SPW}}=289 \mathrm{kN} \mathrm{m}^{-2}\right.$; Lou et al. 2002). Specific tension values specifically for holocephalan red and white muscles are not available. Therefore, elasmobranch values were used to model muscle force for the anterior and posterior divisions of the adductor mandibulae.

In-lever distances were determined for each muscle from the three-dimensional coordinates of their respective insertions on the lower jaw and the jaw joint. A weighted average of these in-levers was then estimated based on the forces produced by their respective muscles. Out-lever distances for biting at the anterior and posterior margins of the tooth plate on the lower jaw were determined from the three-dimensional coordinates of these bite points and the jaw joint (figure $2 b$ ). MA ratios for anterior and posterior biting were then calculated by dividing the resolved in-lever by the out-levers for anterior and posterior biting.

Force vectors were created for each muscle based on their theoretical maximum tetanic forces and threedimensional coordinates of the origin and insertion. These vectors were reflected about the $X-Y$ plane to represent forces generated bilaterally by the jawadducting musculature. Theoretical maximum anterior and posterior bite forces were then estimated in H. colliei using MATHCAD v. 11.1 software (Mathsoft, Inc., Cambridge, MA, USA) by summing the threedimensional moments generated by the jaw-adducting musculature about the jaw joints. The static equilibrium conditions for the forces acting on the lower jaw are

$$
\sum F_{\mathrm{LJ}}=F_{\mathrm{AMA}-\alpha}+F_{\mathrm{AMA}-\beta}+F_{\mathrm{AMP}}+F_{\mathrm{B}}+F_{\mathrm{JR}}=0,
$$

where $F_{\mathrm{AMA}-\alpha}$ and $F_{\mathrm{AMA}-\beta}$ are the forces generated by the adductor mandibulae anterior- $\alpha$ and $-\beta$, respectively; $F_{\mathrm{AMP}}$ is the force generated by the adductor mandibulae posterior; $F_{\mathrm{B}}$ is the bite reaction force from a prey item and is equal and opposite to bite force; and $F_{\mathrm{JR}}$ is the jaw joint reaction force. Joint reaction forces (JRF) maintain the static equilibrium of feeding mechanisms by balancing the moments acting upon the jaws via their associated musculature and contact with prey items.

\subsection{Tetanic bite force}

Hydrolagus colliei were anaesthetized with MS-222 $\left(0.133 \mathrm{~g} \mathrm{l}^{-1}\right)$, after which the $\alpha$ and $\beta$ subdivisions of the adductor mandibulae anterior (figure $2 a$ ) were bilaterally implanted with a unipolar electrode in the form of 24-gauge stainless steel hypodermic needles connected to an SD9 stimulator (Grass Telefactor, West Warwick, RI, USA). The posterior adductor division was too small to be reliably implanted. Tetanic contraction of the adductor mandibulae anterior was accomplished via stimulation $(10 \mathrm{~V}, 100 \mathrm{~Hz}, 0.02 \mathrm{~ms}$ delay, $3 \mathrm{~ms}$ pulse width, $1 \mathrm{~s}$ duration), while a piezoelectric load cell (Model 201B02, PCB Piezotronics, Inc., 
Depew, NY, USA) was placed between the anterior tips of the upper and lower tooth plates providing an empirical test of our theoretical calculations of anterior bite force. Bite force $(\mathrm{N})$ data were acquired with a $6020 \mathrm{E}$ data acquisition board and LABVIEW v. 6.0 software (National Instruments Corp., Austin, TX, USA). The load cell was fitted with lever arms, the distance between which was adjusted to ensure that all individuals over the size range investigated bit the device at approximately the same acute gape angle. The small gape made it impossible to place the transducer at the posterior bite point, precluding a comparison of theoretical and in vivo estimates at this position. Three bites were recorded in each trial, after which the animals were returned to the holding tank. Animals were perfused with aerated seawater between each bite during a given trial. Trials were repeated on no more than three consecutive days until maximum bite forces were consistent from trial to trial.

Dietary analyses suggest that $H$. colliei may be a benthic grazer, opportunistically consuming any locally abundant prey item (Wingert et al. 1979; Quinn et al. 1980). To determine whether $H$. colliei is capable of repeatedly generating high bite forces or whether muscle fatigue will limit its ability to graze benthic prey, a bite force fatigue experiment was performed. Muscle stimulation and data acquisition were performed on three H. colliei (37-44 cm SL) in the manner described above. The stimulation protocol for this experiment consisted of 25 individual stimulations, each separated by a $1 \mathrm{~s}$ delay ( 25 stimulations per $50 \mathrm{~s}$ ). The same stimulation protocol was performed on the quadratomandibularis and preorbitalis muscles of three S. acanthias, which were used for comparison in this analysis because their diet of soft-bodied piscine and cephalopod prey does not apparently require sustained or repeatedly high bite forces (Huber \& Motta 2004).

\subsection{Statistical analysis}

Cranial morphometrics, muscle cross-sectional areas, lever measurements, MAs and anterior and posterior bite forces were log transformed and linearly regressed against $\log$ standard length. Scaling relationships of these variables with respect to standard length were determined using a Student's $t$-test in which the slope of a given variable relative to log-transformed standard length was compared with the appropriate isometric slope ( 0, MAs; 1 , cranial morphometrics and lever arm measurements; 2, areas and forces; Zar 1999). Modified $t$-tests were also used to compare the slopes of theoretically estimated anterior bite forces and bite forces measured during tetanic stimulation of the adductor musculature. Linear regression was used to determine the predictive ability of head width and height with respect to bite force in $H$. colliei. Lastly, maximum bite forces and body masses from 14 species of fishes were compiled from the available literature (Hernandez \& Motta 1997; Clifton \& Motta 1998; Huber \& Motta 2004; Korff \& Wainwright 2004; Huber et al. 2005, 2006; Huber 2006). When species data were available for a range of sizes, mean bite forces and masses were calculated. Bite forces and body masses were $\log$ transformed and mass-specific bite forces were determined from the studentized residuals of a linear regression of $\log$ bite force versus log body mass. Residuals were qualitatively compared to determine the ranges of relative bite forces of durophagous and non-durophagous chondrichthyans and bony fishes. Dietary categorizations were based upon the literature from which the bite force data were compiled. In the comparative portion of this study, we are primarily interested in characterizing performance parameters of durophagous fishes; as a result, and as there is a wide variation in the fine-scale resolution of the phylogenies of these fishes, values were not scaled phylogenetically (e.g. accounting for branch lengths). The $t$-tests for scaling analyses were performed manually with a $p$-value of 0.05 . All other statistical analyses were performed in SYSTAT v. 10.0 with a $p$-value of 0.05 (SYSTAT Software, Inc.).

\section{RESULTS}

\subsection{Scaling of feeding biomechanics}

The cross-sectional areas of the adductor mandibulae anterior- $\alpha$, adductor mandibulae anterior- $\beta$ and adductor mandibulae posterior scaled isometrically over the ontogenetic series of $H$. colliei (table 1). Both the mean fibre angle and mean fibre length of the anterior- $\alpha$ and $-\beta$ divisions increased over ontogeny (figure 3). However, variability among these measures and muscle masses resulted in isometric growth of each muscle's physiological cross-sectional area. The adductor mandibulae anterior- $\beta$ theoretically generated the most force at all sizes (51\%), followed by the adductor mandibulae anterior- $\alpha(42 \%)$ and adductor mandibulae posterior (7\%).

Although the relative force-producing capacity of the cranial musculature did not change over ontogeny, the lever mechanics of the feeding mechanism changed substantially. The resolved in-lever for jaw adduction scaled with positive allometry $(a=1.25)$, while the out-levers to both the anterior and posterior margins of the mandibular tooth plate scaled with negative allometry (anterior out-lever: $a=0.83$; posterior outlever: $a=0.63$; table 1 ). Consequently, positive allometry was observed in the MA of the feeding mechanism at both the anterior $(a=0.41)$ and posterior $(a=0.61)$ bite points (table 1 ; figure 4$)$. Anterior MA ranged from 0.48 to 0.68 , while posterior MA ranged from 1.12 to 1.91 over the ontogenetic series of $H$. colliei (figure $4 b$ ).

Given a relatively constant magnitude of force produced by the cranial musculature relative to standard length (isometric muscle cross-sectional area) and an allometric increase in the MA of the feeding mechanism, both anterior (11-104 N) and posterior (23-191 N) theoretical bite forces increased with positive allometry over ontogeny (anterior bite force: $a=3.13$; posterior bite force: $a=3.12$ ). Despite the anatomical evidence supporting hyperallometry of performance, tetanically stimulated anterior bite force $(10-58 \mathrm{~N})$ scaled isometrically over ontogeny $(a=2.34$; table 1 ; figure 5$)$. Although a discrepancy was observed between these scaling patterns, the regression slopes of tetanically 
Table 1. Results of the regressions of standard length against biomechanical variables in the feeding mechanism of $H$. colliei. (Bonferroni corrected $p=0.0038$.)

\begin{tabular}{|c|c|c|c|c|c|}
\hline \multirow[b]{2}{*}{ variable } & \multicolumn{2}{|c|}{$\begin{array}{l}\text { regression equation } \\
(\log Y=a \log \mathrm{SL}+b)\end{array}$} & \multirow[b]{2}{*}{$r^{2}$} & \multirow[b]{2}{*}{$t$-score $(6)$} & \multirow[b]{2}{*}{$p$-value } \\
\hline & slope $(a)$ & intercept $(b)$ & & & \\
\hline head width $(\mathrm{cm})$ & 0.80 & -0.65 & 0.93 & 2.280 & 0.0314 \\
\hline head height $(\mathrm{cm})$ & 0.86 & -0.54 & 0.97 & 2.456 & 0.0247 \\
\hline adductor mandibulae anterior- $\alpha \mathrm{CSA}\left(\mathrm{cm}^{2}\right)$ & 2.32 & -3.80 & 0.95 & 1.409 & 0.1042 \\
\hline adductor mandibulae anterior- $\beta$ CSA $\left(\mathrm{cm}^{2}\right)$ & 2.37 & -3.79 & 0.92 & 1.347 & 0.1133 \\
\hline adductor mandibulae posterior $\operatorname{CSA}\left(\mathrm{cm}^{2}\right)$ & 1.69 & -3.62 & 0.82 & 0.949 & 0.1896 \\
\hline in-lever $(\mathrm{cm})$ & 1.25 & -1.79 & 0.96 & 2.492 & 0.0235 \\
\hline anterior out-lever $(\mathrm{cm})$ & 0.83 & -0.90 & 0.95 & 2.285 & 0.0312 \\
\hline posterior out-lever $(\mathrm{cm})$ & 0.63 & -1.02 & 0.71 & 2.276 & 0.0316 \\
\hline anterior mechanical advantage & 0.41 & -0.87 & 0.60 & 2.993 & 0.0121 \\
\hline posterior mechanical advantage & 0.61 & -0.76 & 0.50 & 2.468 & 0.0243 \\
\hline electrically stimulated anterior bite force $(\mathrm{N})$ & 2.34 & -2.06 & 0.74 & 0.593 & 0.2874 \\
\hline theoretical anterior bite force $(\mathrm{N})$ & 3.13 & -3.09 & 0.99 & 9.397 & $<0.0001$ \\
\hline theoretical posterior bite force $(\mathrm{N})$ & 3.12 & -2.74 & 0.97 & 5.105 & 0.0011 \\
\hline
\end{tabular}

stimulated and theoretical anterior bite force were not statistically different. The statistical equivalence of slopes is probably due to the greater variability in tetanically stimulated bite force measurements $\left(r^{2}=0.74\right)$ than in theoretical anterior bite force $\left(r^{2}=0.99 ;\right.$ table 1$)$. Regardless of whether the small adductor mandibulae posterior was included in the model, theoretical anterior bite force was always greater than tetanically stimulated anterior bite force (figure 6).

Both head width and height scaled with negative allometry in $H$. colliei (table 1 ). Head width and height more accurately predicted theoretical anterior bite force $\left(r^{2}=0.88\right.$ and 0.96 , respectively) than tetanically stimulated bite force $\left(r^{2}=0.63\right.$ and 0.76 , respectively). Head height was a more accurate predictor of anterior bite force than head width for both theoretical and tetanically stimulated methods of determining bite force.

JRF for anterior biting in $H$. colliei increased over ontogeny from 6 to $17 \mathrm{~N}$ (figures 6 and 7 ). The resolved vector of JRF indicates the mode of joint loading during biting. During anterior biting, the joint reaction force was locally oriented anteroventrally relative to the articular surface of the lower jaw and posterodorsally relative to that of the upper jaw, indicating global compression in the jaw joint. The ratio of joint reaction force to bite force for anterior biting decreased with increasing standard length (1.1-0.2), indicating a more even distribution of forces throughout the feeding mechanisms of larger specimens.

JRF for posterior biting also increased in magnitude over ontogeny $(\mid-2$ to $-50 \mid \mathrm{N})$, but were oriented anterodorsally relative to the lower jaw indicating tensile joint loading (figures 6 and 7 ). The ratio of joint reaction force to bite force increased in magnitude over ontogeny for posterior biting as well $(\mid-0.2$ to $-0.5 \mid)$, indicating a disproportionate increase in tensile joint loading in larger specimens, and a potentially greater risk of jaw dislocation and soft tissue damage. Relative changes in force distributions within the feeding mechanism of $H$. colliei are directly attributed to changes in MA (discussed in §3.2).
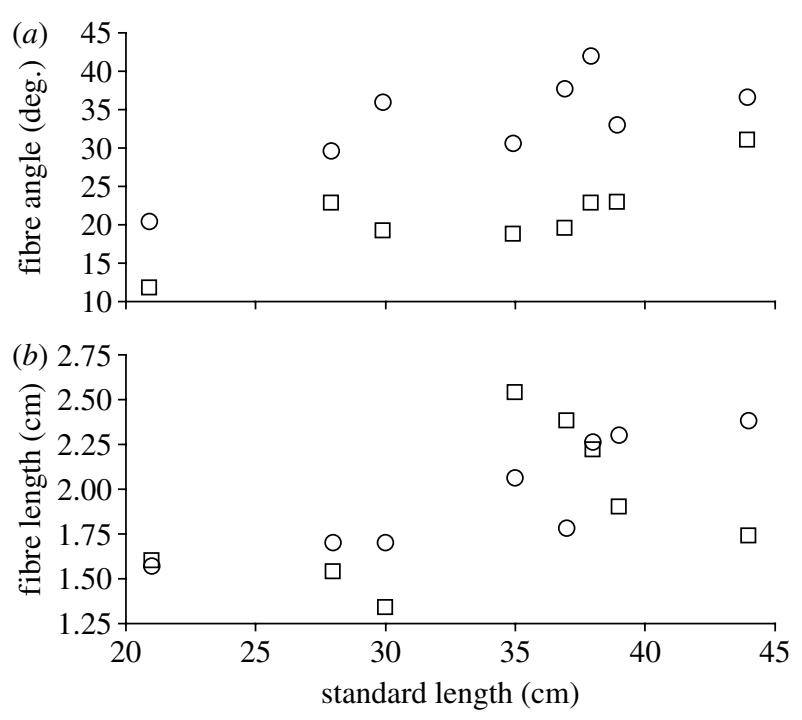

Figure 3. (a) Ontogenetic changes in the fibre angles of the adductor mandibulae anterior- $\alpha$ (squares) and adductor mandibulae anterior- $\beta$ (circles) subdivisions with respect to standard length in $H$. colliei. (b) Ontogenetic changes in the fibre lengths of the adductor mandibulae anterior- $\alpha$ (squares) and adductor mandibulae anterior- $\beta$ (circles) subdivisions with respect to standard length in $H$. colliei. Values of $r^{2}$ for associated regression equations can be found in table 1 .

\subsection{Comparative feeding biomechanics}

Dietary analyses indicate that $H$. colliei may graze benthic prey, which would be facilitated by the fatigueresistant jaw musculature. However, the principal task of generating high bite forces for crushing hard prey was hypothesized to cause rapid muscle fatigue. Tetanically stimulated anterior bite force of $H$. colliei decreased by $85 \%$ at a rate of $3.3 \%$ per stimulation during the jaw adductor fatigue trials. In the non-durophagous spiny dogfish $S$. acanthias, tetanically stimulated anterior bite force decreased by $50 \%$ at a rate of $2.0 \%$ per stimulation (figure 8). It should be noted that the redfibred adductor mandibular posterior, which may play 


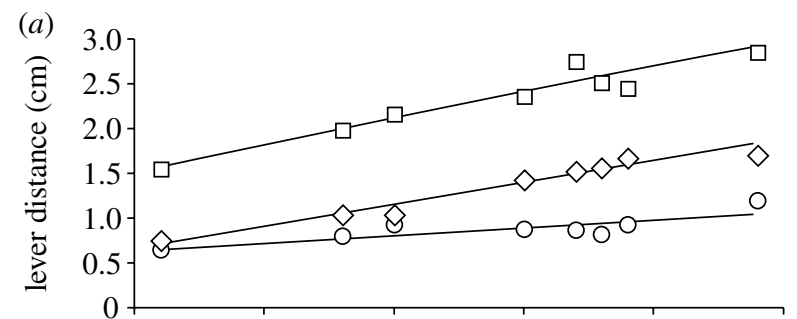

(b)

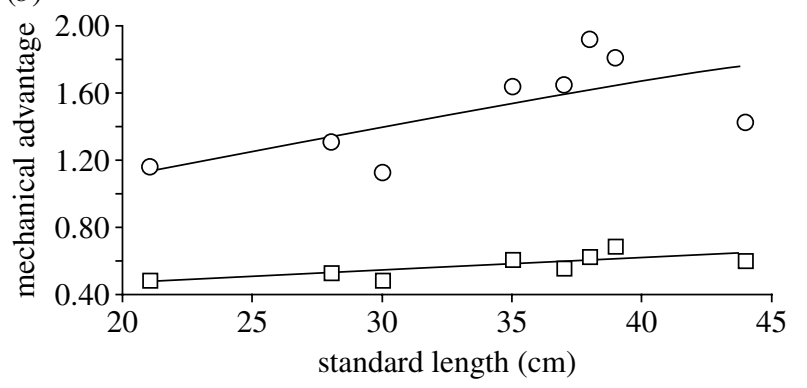

Figure 4. (a) Ontogenetic changes among in-lever (diamonds) and anterior (squares) and posterior (circles) out-lever distances associated with jaw adduction in $H$. colliei with respect to standard length. The resolved in-lever scaled with positive allometry, while both out-levers scaled with negative allometry. (b) Ontogenetic changes in anterior (squares) and posterior (circles) MAs for jaw adduction in $H$. colliei with respect to standard length. MA scaled with positive allometry at both the anterior and posterior bite points. Values of $r^{2}$ for associated regression equations can be found in table 1 .

a role in fatigue resistance, was not stimulated in H. colliei. However, its minimal contribution to the adductive force $(7 \%)$ suggests that it will not play a significant role.

The comparative analysis of bite forces revealed that $H$. colliei produces one of the most forceful bites relative to body mass for those fishes in which bite force has been determined (table 2). The only fishes with higher mass-specific bite forces are the striped burrfish (Chilomycterus schoepfi), hogfish (Lachnolaimus maximus), sheepshead (Archosargus probatocephalus) and sharpnose sevengill shark (Heptranchias perlo), the first three of which are durophagous. Within the chondrichthyan fishes, the mass-specific bite force of $H$. collie $i$ is only surpassed by $H$. perlo. Excluding the carnivorous $H$. perlo and durophagous slippery dick Halichoeres bivittatus, all of the durophagous fishes in the analysis had higher mass-specific bite forces than those species with non-durophagous diets (table 2).

\section{DISCUSSION}

\subsection{Crushing mechanics of holocephalans}

Our data show that the spotted ratfish, though their jaws are not heavily mineralized, can generate massspecific bite forces equivalent to those of hard preycrushing elasmobranchs and therefore are capable of eating similarly hard prey. In the absence of skeletal reinforcement of the jaws and apparent modifications to muscular physiology, we conclude that this highperformance capability is achieved by mechanically favourable geometries of the feeding mechanism.

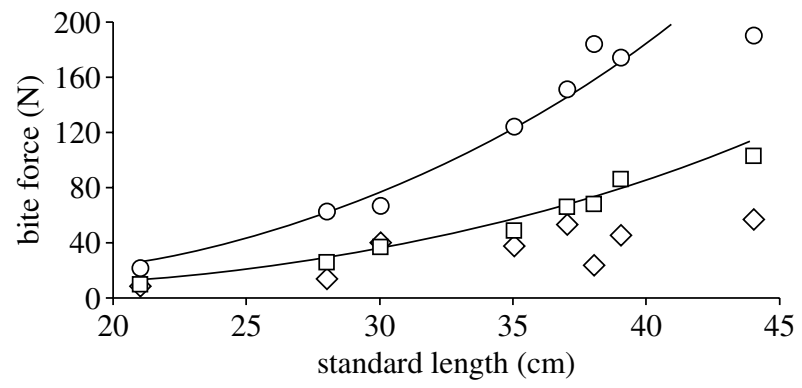

Figure 5. Ontogenetic changes in the bite force of $H$. colliei with respect to standard length as determined by theoretical biomechanical modelling (squares, anterior; circles, posterior) and tetanic muscle stimulation experiments (diamonds, tetanic). Both anterior and posterior theoretical bite forces scaled with positive allometry, while tetanically stimulated bite force scaled isometrically. Values of $r^{2}$ for associated regression equations can be found in table 1 .

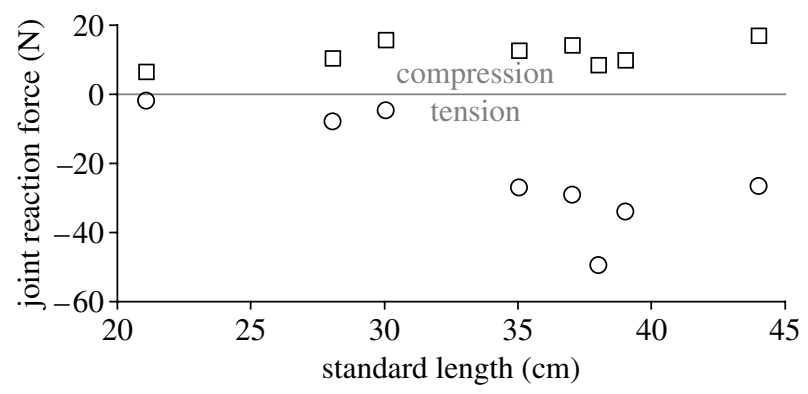

Figure 6. Ontogenetic changes in JRF with respect to standard length in $H$. colliei for anterior (squares) and posterior (circles) biting. Positive values indicate compressive JRF associated with anterior biting, during which MA for jaw adduction is less than 1.0. Negative values indicate tensile (and potentially destabilizing) JRF associated with posterior biting, during which MA for jaw adduction is greater than 1.0.

Jaw closure is effected by the three divisions of the adductor mandibulae. The two anterior divisions insert at approximately 50-70\% along the length of the jaw, while the posterior division inserts nearly on the jaw joint. The two pinnate-fibred subdivisions of the anterior adductor appear to be forceful jaw closers. These divisions were implanted for the stimulation experiments and, as we expect from high forcegenerating white-fibred muscles, they fatigue 1.6 times more rapidly than similar muscles from a nondurophagous cartilaginous fish. The posterior division is red-fibred and though we might expect that it is involved in stabilizing the jaw joint, our model shows that it has a negligible contribution to bite force and the reduction in tensile JRF during posterior biting. Although the posterior division may facilitate rapid jaw adduction via its low MA, we hypothesize it is more involved in facilitating benthic grazing by virtue of its fatigue-resistant, red fibre composition.

Our model illustrates that joint reaction force and MA are mechanically interrelated. MA, the ratio of in-lever to out-lever length, represents the proportion of muscular force transferred to the crushing surface of the jaws. JRF balance the difference between input force and bite force, and are therefore representative of the stability of the system. A MA of 1.0 indicates that the 
(a)
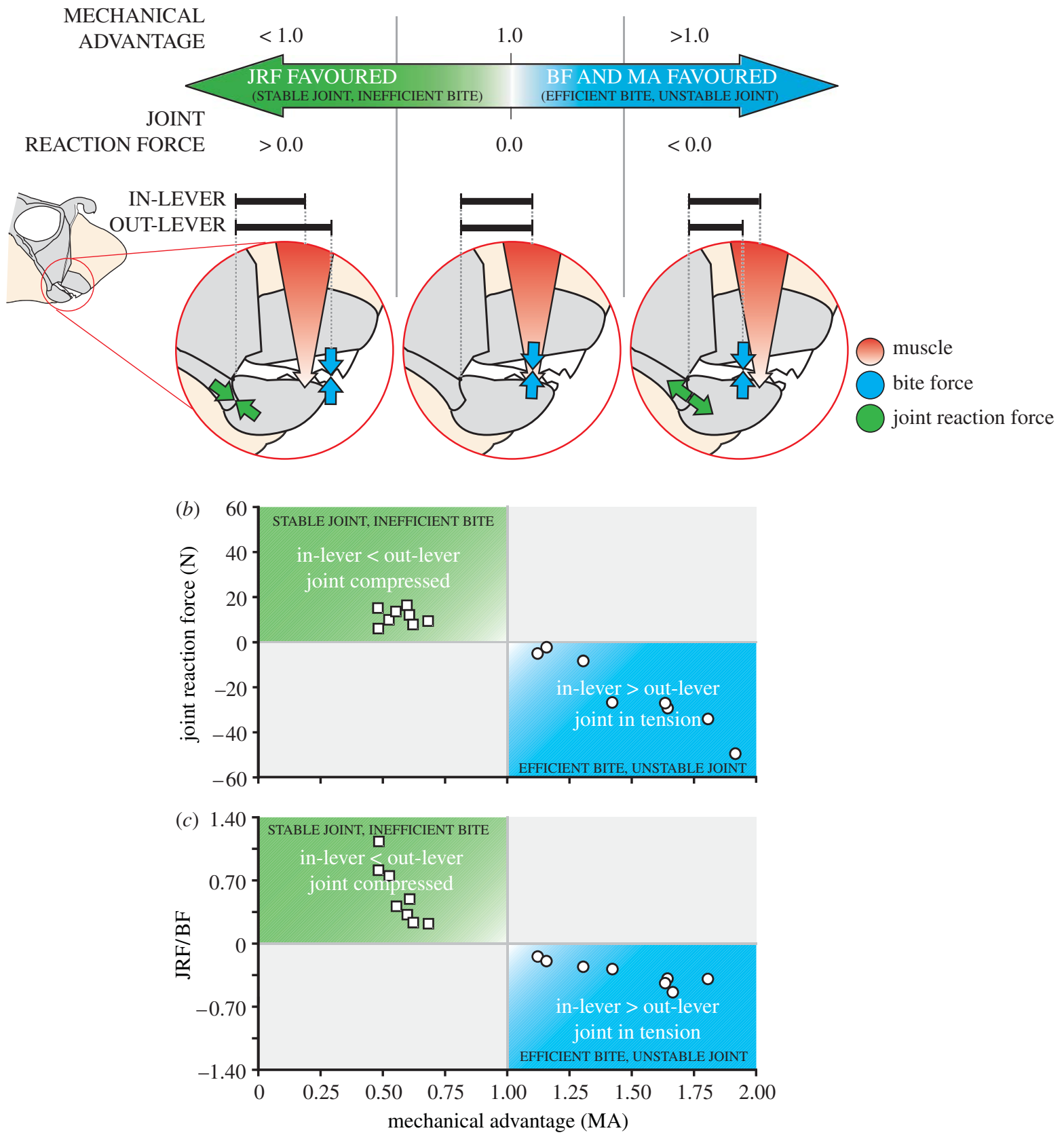

Figure 7. (a) Graded arrow represents the trade-off between the stability of the jaw joint and the efficiency of force transfer from the jaw adductor musculature to prey as a function of MA. When MA is less than 1.0 (left inset of the jaws of $H$. colliei), less than $100 \%$ of the muscular force is transmitted as bite force (BF) and the jaw joint is stabilized (favoured) via compressive JRF. When MA is greater than 1.0 (right inset), bite force exceeds the adductive muscular force via force amplification of the mandibular lever system. However, the prey item acts as a temporary fulcrum about which the lower jaw rotates, resulting in tensile JRF that destabilize the joint. When MA is 1.0 (centre inset), the lower jaw is in static equilibrium and JRF do not occur. (b) Magnitude and orientation of JRF in H. colliei as a function of MA. (c) Ratio of joint reaction force to bite force as a function of MA in $H$. colliei. As MA increases to 1.0, more of the adductive muscular force is transferred to the prey (increasing bite force) and less force must be balanced at the jaw joint, resulting in low ratios. When MA is greater than 1.0, bite force exceeds the adductive muscular force and the difference of these forces must be balanced at the jaw joint. In either case, low ratios indicate reduced joint loading. In $(b, c)$ positive values indicate joint-stabilizing compressive forces that occur when MA is less than 1.0, whereas negative values indicate joint-destabilizing tensile forces that occur when MA is greater than 1.0 (squares, anterior; circles, posterior).

in- and out-levers are the same, as are the input and output forces. In this case, the joint reaction force is 0.0 and the joint is unloaded. As MA decreases from 1.0 (i.e. as the in-lever becomes shorter than the out-lever), the crushing force is less than the input force but the joint is stabilized by compressive loading. However, when MA exceeds 1.0, the in-lever is farther from the fulcrum than the out-lever, the crushing force is greater than the input force, and the joint is destabilized by tensile loading (figure 7).

During anterior biting in $H$. colliei, MA is less than 1.0 and compressive loading stabilizes the jaw joint. However, the posterior margin of the tooth plates is closer to the jaw joint than the adductor insertion, 


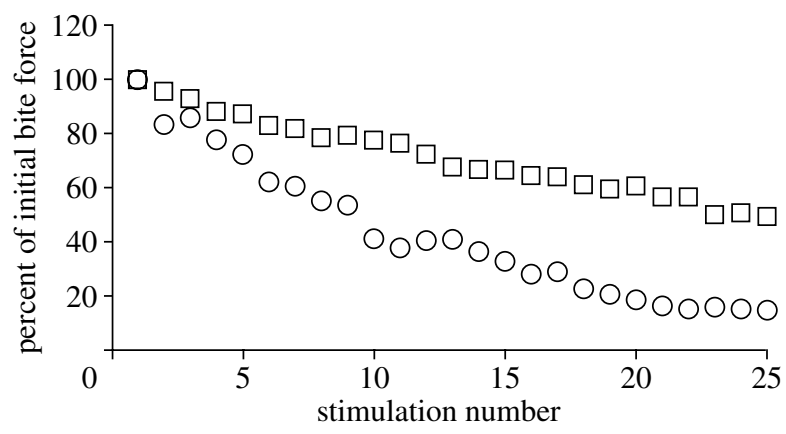

Figure 8. Incremental decrease in bite force with repeated tetanic stimulation of the jaw-adducting musculature in $H$. colliei (circles) and S. acanthias (squares). As expected of high force-producing muscles, the jaw adductors of H. colliei fatigued rapidly.

resulting in a MA greater than 1.0 and an amplification of input force. While this facilitates the crushing of hard prey, it also represents a trade-off in the stability of the feeding mechanism as it causes tensile JRF. Hydrolagus colliei may function between the two extremes of low MA/joint stabilization and high MA/joint destabilization. Wear patterns on the notch in the mandibular tooth plate suggest that prey is sheared just anterior to the resolved in-lever where MA is slightly less than 1.0. This location has a high MA and biting at this point will cause compressive rather than tensile joint loading, avoiding potential joint dislocation or soft tissue damage (figure 7 ).

\subsection{Ontogeny of feeding biomechanics}

As $H$. colliei doubles in length, the measured ability to exert crushing force increases sixfold and theoretically calculated forces increase ninefold. This increase in performance can be accomplished by allometric increases in muscle force or MA, and both are often implicated (Hernandez \& Motta 1997; Wainwright \& Shaw 1999). The piscivorous blacktip shark Carcharhinus limbatus has a relative increase in bite force over ontogeny owing to hyperallometric growth in both the adductor musculature and the leverage system (Huber et al. 2006). In contrast, positive allometry of bite force in $H$. collie $i$ can be attributed to changes in the MA of the feeding mechanism because the jaw musculature grows isometrically. Regardless of the underlying developmental mechanism, recent studies suggest that hyperallometric increases in bite force may be a general phenomenon among vertebrates because high bite force is correlated with increased prey handling efficiency and reduced trophic energy expenditure (Herrel et al. 2001; Verwaijen et al. 2002; Herrel \& Gibb 2006).

We suggest that isometry of muscle physiological cross-sectional area may be indicative of a constraint on available cranial volume. If the head and jaw musculature of $H$. colliei grew isometrically, we would expect the force generated by the jaw muscles to also scale isometrically. However, as H. colliei grows, its head becomes narrower and shallower, leaving less room for the jaw adductors and inhibiting isometric growth of anatomical cross-sectional area. The solution to getting more force from a given volume and anatomical cross section of muscle is to shift to a pinnate fibre organization, which increases the physiological cross-sectional area. This has been observed in the blacktip shark $C$. limbatus, in which two divisions of the main adductor shift from parallel to pinnate architecture at approximately $90 \mathrm{~cm}$ TL (Huber et al. 2006). As we might expect from a volume-constrained system, the pinnation angles of the anterior adductor subdivisions increase sharply as $H$. colliei grows, allowing isometric force generation from a hypoallometric cranial volume. We were, however, surprised to find an ontogenetic increase in mean fibre length, which simultaneously decreases physiological cross-sectional area (equation (2.2)).

Although the biomechanical model used in this analysis has accurately predicted maximum bite forces in numerous cartilaginous fishes (Huber \& Motta 2004; Huber et al. 2005; Huber 2006), theoretical maximum anterior bite force was significantly greater than tetanically stimulated anterior bite force. Hypothetically, this discrepancy could be due to a difference between the specific tensions of holocephalan and elasmobranch muscles, the latter of which was used to calculate maximum tetanic forces of the jaw adductors in H. colliei. However, this would not explain the increasing divergence between theory and measured forces as the animal grows. Other explanations could scale with size though - total force generated by the muscle might not be measured at the jaws owing to losses due to pliancy of the cartilaginous cranial skeleton or looseness in the joints of the jaw. Alternatively, ontogenetic changes in the contractile dynamics of these muscles could reduce the accuracy of the theoretical model (Richard \& Wainwright 1995). A less satisfying explanation is that there is incomplete stimulation of the feeding muscles in the larger animals. We suppose that our unipolar stimulation electrodes would make this scenario unlikely, but losses of this sort would certainly scale with size.

\subsection{Comparative feeding biomechanics}

MA for anterior biting in $H$. colliei $(0.48-0.68)$ is higher than that of most fishes, even at the smallest sizes sampled (Huber \& Motta 2004; Wainwright et al. 2004; Westneat 2004; Huber et al. 2005, 2006). The only other chondrichthyan fish with a comparable MA is the durophagous horn shark $H$. francisci (0.51), which is surpassed early in the ontogeny of $H$. colliei (approx. $28 \mathrm{~cm}$ TL; figure 4). Among actinopterygian fishes, only the durophagous parrotfishes (Scaridae) have higher jaw-adducting MAs than $H$. colliei (Wainwright et al. 2004; Westneat 2004).

High mass-specific bite forces were commonly associated with durophagy among the fishes included in this analysis. Seven of the eight largest mass-specific bite forces were from durophagous taxa, with only a single durophagous species exhibiting mass-specific bite forces similar to those of non-durophagous species (slippery dick $H$. bivittatus). Similar ecobiomechanical correlations have been found between jaw mechanics and diet in numerous teleosts (Westneat 1995, 2004). The only chondrichthyan with a higher mass-specific 
Table 2. Mass-specific analysis of bite forces among fishes.

\begin{tabular}{|c|c|c|c|c|}
\hline species & common name & $\begin{array}{l}\text { anterior bite } \\
\text { force }(\mathrm{N})\end{array}$ & $\operatorname{mass}(\mathrm{g})$ & $\begin{array}{l}\text { residual bite } \\
\text { force }\end{array}$ \\
\hline Chilomycterus schoepfi ${ }^{\mathrm{a}}$ & striped burrfish & 380 & 180 & 2.11 \\
\hline Lachnolaimus maximus ${ }^{\mathrm{a}, \mathrm{b}}$ & hogfish & 290 & 209 & 1.78 \\
\hline Archosargus probatocephalus ${ }^{\mathrm{a}}$ & sheepshead & 186 & 581 & 0.87 \\
\hline Heptranchias perlo & sharpnose sevengill & 132 & 1115 & 0.21 \\
\hline Hydrolagus colliei ${ }^{\mathrm{a}}$ & spotted ratfish & 69 & 452 & 0.05 \\
\hline Heterodontus francisci $i^{\mathrm{a}}$ & horn shark & 148 & 2604 & -0.11 \\
\hline Halichoeres maculipinna ${ }^{\mathrm{a}, \mathrm{b}}$ & clown wrasse & 11 & 18 & -0.13 \\
\hline Halichoeres garnoti ${ }^{\mathrm{a}, \mathrm{b}}$ & yellowhead wrasse & 10 & 21 & -0.29 \\
\hline Chiloscyllium plagiosum & white-spotted bamboo shark & 69 & 1041 & -0.38 \\
\hline Thalassoma bifasciatum $^{\mathrm{b}}$ & bluehead wrasse & 5 & 7 & -0.43 \\
\hline Negaprion brevirostris & lemon shark & 69 & 1309 & -0.49 \\
\hline Carcharhinus limbatus & blacktip shark & 104 & 5618 & -0.89 \\
\hline Halichoeres bivittatus ${ }^{\mathrm{a}, \mathrm{b}}$ & slippery dick & 5 & 19 & -0.98 \\
\hline Squalus acanthias & spiny dogfish & 12 & 408 & -1.57 \\
\hline
\end{tabular}

${ }^{\mathrm{a}}$ Diet is predominantly (more than 50\%) durophagous.

${ }^{\mathrm{b}}$ Pharyngeal bite force.

bite force than $H$. collie $i$ was surprisingly the piscivorous sharpnose sevengill shark $H$. perlo, whose high bite force is a function of disproportionately large jaw adductors occupying deep fossae in the upper and lower jaws, a characteristic of the ancestral cranial morphotype for sharks (Compagno 1977; Huber 2006). This apparent 'overbuilding' of the adductor for a piscivorous lifestyle suggests that other selective pressures may have shaped the feeding mechanisms of basal sharks such as H. perlo. Most non-durophagous chondrichthyans had low mass-specific bite forces, suggesting that factors such as dentition or velocity of jaw adduction may figure prominently in their ability to capture prey. The piscivorous spiny dogfish $S$. acanthias had the lowest mass-specific bite force and has a correspondingly low jaw-adducting MA (0.28) associated with capturing elusive prey (Huber \& Motta 2004).

While mass-specific bite force is a relative indicator of the ability to consume hard prey, absolute bite force will determine whether a given prey item can be consumed. The threshold forces required to crush prey exoskeletons represent minimum performance requirements that separate durophagous and non-durophagous species. This performance determinant may serve as the selective pressure that results in the positive allometry of bite force seen in this study, allowing $H$. colliei to consume hard prey earlier in its life history than an isometric ontogenetic trajectory. Hyperallometry of feeding performance may reduce net trophic energy expenditure as well by permitting the consumption of relatively large prey that have higher fracture thresholds and nutritional value than small prey (Herrel et al. 2001; Korff \& Wainwright 2004).

\subsection{Holocephalan versus elasmobranch durophagy}

We propose that a comparison between the anatomies of durophagous holocephalan and elasmobranch feeding systems highlights, in general, those characters that are functionally important in the crushing of hard prey.
However, our data suggest that there are several striking differences between the feeding mechanisms of these groups. The holocephalan feeding mechanism is poorly, if at all, calcified, contains a vaulted ethmoidal region of the cranium to which the upper jaw is fused (autostylic jaw suspension), a short and ventrally directed lower jaw and densely mineralized tooth plates that are never replaced. Conversely, the elasmobranch feeding mechanism is tessellated ('tiled' with calcified tissue) and consists of a relatively shallow cranium, large, kinetic jaws that articulate with the cranium via cartilaginous and ligamentous attachments (hyostylic jaw suspension), and rapid serial replacement of individual teeth. The divergence of holocephalans and elasmobranchs began in the Devonian period and both the lineages had diversified considerably in the Carboniferous period. Our data from a single, extant holocephalan species allow little insight into the shaping of the lineage, yet our demonstration that these fishes are capable of generating high bite forces does support the hypothesis that the holocephalan transition to autostyly, vaulting of cranial geometry and the reduction/ fusion of dental elements is a product of selection for durophagy in the evolution of the holocephalan cranial morphotype (Grogan \& Lund 2004).

The characteristic expanded holocephalan tooth plates serve to spread crushing forces across the entire surface of the jaws, perhaps eliminating the need for mineralization at any individual bite point. In the absence of heavy mineralization, the vaulted cranial skeleton and fused upper jaw of holocephalans may stabilize the feeding mechanism against dorsoventral flexion. Deformation of the feeding mechanism is a particular concern for durophagous taxa owing to the large reaction forces that occur when consuming hard prey. Dorsoventral flexion of the cranium would dampen these reaction forces and reduce the ability of $H$. colliei to fracture its prey. However, negative allometry of head depth may represent an ontogenetic strategy for durophagy in $H$. colliei early in its life history. Relatively greater vaulting of the cranium in 
younger individuals may provide greater resistance to flexion, whereas the damping of reaction forces is a less significant problem for larger individuals with higher absolute bite forces.

Notable differences exist in the cranial musculature of durophagous holocephalans and elasmobranchs as well. The primary jaw adductor of $H$. collie $i$ is bipinnate and inserts upon the lower jaw via a stout tendon. Cartilage has been proposed to have a low pull-out strength, generally precluding the existence of tendinous point insertions between muscles and skeletal elements; parallel-fibred muscles with aponeurotic surface insertions are typical of the elasmobranch fishes (Liem \& Summers 1999; Summers et al. 2003). The holocephalan adductor mandibulae anterior appears to have circumvented this constraint by inserting on the lower jaw via a tendon sling that wraps beneath the jaw bilaterally, providing significant strain distribution for the high forces produced by the pinnate-fibred divisions of the adductor (Dean et al. 2007a).

\section{CONCLUSIONS}

Despite the substantial morphological disparity among durophagous holocephalans and elasmobranchs, several commonalities have emerged from this analysis. A molariform dentition and fused symphysis are key characteristics for stress distribution and force transfer in the mandibular lever system. High force production by the jaw adductors, via a pinnate or parallel fibre architecture, and high jaw leverage have convergently evolved in these taxa as well. The emergent property of these biomechanical characteristics is high feeding performance among durophagous cartilaginous fishes relative to non-durophagous ones. We suggest that this suite of characters is highly selected for in durophagous cartilaginous fishes. Positive allometry of bite force in $H$. colliei was attributed to a suite of muscular and skeletal characteristics, all facilitating an ability to crush hard prey early in its life history. The feeding mechanism of $H$. colliei thoroughly illustrates the trade-off between joint stability and adductor force transmission during feeding as well. We expect that biomechanical characteristics associated with crushing hard prey that were identified in this study will be shared widely among durophagous holocephalans.

All husbandry and experimentation were performed in accordance with the guidelines of the Institutional Animal Care and Use Committee of the University of Washington.

We would like to thank Craig Staude and the staff of the University of Washington Friday Harbor Laboratories for dedicating their time, energy and resources to this project. J. Schaefer and M. Porter provided valuable experimental assistance, and P. Motta provided much insight during the development of this manuscript. We would also like to thank the Biology Departments of the University of Tampa, the University of California Irvine and the University of South Florida for their continual support. This research was supported by a National Science Foundation grant to M.N.D. and A.P.S. (IOB-0616322), a University of South Florida Foundation presidential doctoral fellowship to D.R.H. and a University of California Irvine Chancellor's Club fellowship to M.N.D.

\section{REFERENCES}

Clifton, K. B. \& Motta, P. J. 1998 Feeding morphology, diet, and ecomorphological relationships among five Caribbean labrids (Teleostei, Labridae). Copeia 1998, 953-966. (doi:10.2307/1447342)

Compagno, L. J. V. 1977 Phyletic relationships of living sharks and rays. Am. Zool. 17, 303-322.

Compagno, L. J. V. 2005 Checklist of living Chondrichthyes. Reproductive biology and phylogeny, vol. 3 (ed. W. C. Hamlett), pp. 503-548. Enfield, NH: Science Publishers, Inc.

Dean, M. N., Azizi, E. \& Summers, A. P. $2007 a$ Uniform strain in broad muscles: active and passive effects of the twisted tendon of the ratfish Hydrolagus colliei. J. Exp. Biol. 210, 3395-3406. (doi:10.1242/jeb.007062)

Dean, M. N., Bizzarro, J. J. \& Summers, A. P. $2007 b$ The evolution of cranial design, diet, and feeding mechanisms in batoid fishes. Integr. Comp. Biol. 47, 70-81. (doi:10. 1093/icb/icm034)

Didier, D. A. 1995 Phylogenetic systematics of extant chimaeroid fishes (Holocephali, Chimaeroidei). Am. Mus. Novit. 3119, 1-86.

Ebert, D. A. 2003 Sharks, rays, and chimaeras of California. Berkeley, CA: University of California Press.

Grogan, E. D. \& Lund, R. 2004 The origin and relationships of early Chondrichthyes. In Biology of sharks and their relatives (eds J. C. Carrier, J. A. Musick \& M. R. Heithaus), pp. 3-31. Boca Raton, FL: CRC Press.

Hernandez, L. P. \& Motta, P. J. 1997 Trophic consequences of differential performance: ontogeny of oral jaw-crushing performance in the sheepshead, Archosargus probatocephalus (Teleostei, Sparidae). J. Zool. 243, 737-756.

Herrel, A. \& Gibb, A. C. 2006 Ontogeny of performance in vertebrates. Physiol. Biochem. Zool. 79, 1-6. (doi:10.1086/ 498196)

Herrel, A., Van Damme, R., Vanhooydonck, B. \& De Vree, F. 2001 The implications of bite force for diet in two species of lacertid lizards. Can. J. Zool. 79, 662-670. (doi:10.1139/ cjz-79-4-662)

Huber, D. R. 2006 Cranial biomechanics and feeding performance of sharks. In Department of biology, p. 250. Tampa, FL: University of South Florida.

Huber, D. R. \& Motta, P. J. 2004 Comparative analysis of methods for determining bite force in the spiny dogfish Squalus acanthias. J. Exp. Zool. 301A, 26-37. (doi:10. 1002/jez.a.20003)

Huber, D. R., Eason, T. G., Hueter, R. E. \& Motta, P. J. 2005 Analysis of the bite force and mechanical design of the feeding mechanism of the durophagous horn shark Heterodontus francisci. J. Exp. Biol. 208, 3553-3571. (doi:10.1242/jeb.01816)

Huber, D. R., Weggelaar, C. L. \& Motta, P. J. 2006 Scaling of bite force in the blacktip shark Carcharhinus limbatus. Zoology 109, 109-119. (doi:10.1016/j.zool.2005.12.002)

Johnson, A. G. \& Horton, H. F. 1972 Length-weight relationship, food habits, parasites, and sex and age determination of the ratfish, Hydrolagus colliei (Lay and Bennett). Fish. Bull. 70, 421-429.

Korff, W. L. \& Wainwright, P. C. 2004 Motor pattern control for increasing crushing force in the striped burrfish (Chilomycterus schoepfi). Zoology 107, 335-346. (doi:10. 1016/j.zool.2004.09.001)

Liem, K. F. \& Summers, A. P. 1999 Muscular system: gross anatomy and functional morphology of muscles. In Sharks, skates, and rays: the biology of elasmobranch fishes (ed. W. C. Hamlett), pp. 93-114. Baltimore, MD: Johns Hopkins Press. 
Lou, F., Curtin, N. A. \& Woledge, R. C. 2002 Isometric and isovelocity contractile performance of red muscle fibers from the dogfish Scyliorhinus canicula. J. Exp. Biol. 205, $1585-1595$.

Peterson, C. H., Fodrie, F. J., Summerson, H. C. \& Powers, S. P. 2001 Site-specific and density-dependent extinction of prey by schooling rays: generation of a population sink in topquality habitat for bay scallops. Oecologia 129, 349-356.

Powell, P. L., Roy, R. R., Kanim, P., Bello, M. A. \& Edgerton, V. R. 1984 Predictability of skeletal muscle tension from architectural determinations in guinea pigs. J. Appl. Physiol. 57, 1715-1721.

Quinn, T. P., Miller, B. S. \& Wingert, R. C. 1980 Depth distribution and seasonal and diel movements of ratfish, Hydrolagus colliei, in Puget Sound, Washington. Fish. Bull. 78, 816-821.

Richard, B. A. \& Wainwright, P. C. 1995 Scaling the feeding mechanism of largemouth bass (Micropterus salmoides): kinematics of prey capture. J. Exp. Biol. 198, 419-433.

Rome, L. C. 1994 The mechanical design of the fish muscular system. In Mechanics and physiology of animal swimming (eds L. Maddock, Q. Bone \& J. M. V. Rayner), pp. 75-97. Cambridge, UK: Cambridge University Press.

Segura-Zarzosa, J. C., Abitia-Cardenas, L. A. \& GalvanMagana, F. 1997 Observations on the feeding habits of the shark Heterodontus francisci Girard 1854 (Chondrichthyes: Heterodontidae), in San Ignacio Lagoon, Baja California Sur, Mexico. Cienc. Mar. 23, 111-128.

Summers, A. P. 2000 Stiffening the stingray skeleton: an investigation of durophagy in myliobatid stingrays (Chondrichthyes, Batoidea, Myliobatidae). J. Morphol. 243, 113-126. (doi:10.1002/(SICI)1097-4687(200002)243: $2<113:$ :AID-JMOR1 > 3.0.CO;2-A)

Summers, A. P., Koob-Emunds, M. M., Kajiura, S. M. \& Koob, T. J. 2003 A novel fibrocartilaginous tendon from an elasmobranch fish (Rhinoptera bonasus). Cell Tissue Res. 312, 221-227.

Summers, A. P., Ketcham, R. \& Rowe, T. 2004 Structure and function of the horn shark (Heterodontus francisci) cranium through ontogeny: the development of a hard prey crusher. J. Morphol. 260, 1-12. (doi:10.1002/jmor.10141)

Verwaijen, D., Van Damme, R. \& Herrel, A. 2002 Relationships between head size, bite force, prey handling efficiency and diet in two sympatric lacertid lizards. Funct. Ecol. 16, 842-850. (doi:10.1046/j.1365-2435.2002. 00696.x)

Wainwright, P. C. 1988 Morphology and ecology: functional basis of feeding constraints in Caribbean labrid fishes. Ecology 69, 635-645. (doi:10.2307/1941012)

Wainwright, P. C. \& Shaw, S. S. 1999 Morphological basis of kinematic diversity in feeding sunfishes. J. Exp. Biol. 202, 3101-3110.

Wainwright, P. C., Bellwood, D. R., Westneat, M. W., Grubich, J. R. \& Hoey, A. S. 2004 A functional morphospace for the skull of labrid fishes: patterns of diversity in a complex biomechanical system. Biol. J. Linn. Soc. Lond. 82, 1-25. (doi:10.1111/j.1095-8312.2004.00313.x)

Westneat, M. W. 1995 Phylogenetic systematics and biomechanics in ecomorphology. Environ. Biol. Fish. 44, 263-283. (doi:10.1007/BF00005920)

Westneat, M. W. 2004 Evolution of levers and linkages in the feeding mechanisms of fishes. Integr. Comp. Biol. 44, 378-389. (doi:10.1093/icb/44.5.378)

Wingert, R. C., Terry, C. B. \& Miller, B. S. 1979 Food and feeding habits of ecologically important nearshore and demersal fishes in central Puget Sound. Report 7903, University of Washington Fisheries Research Institute.

Zar, J. H. 1999 Biostatistical analysis. Upper Saddle River, NJ: Prentice Hall. 
NOTICE OF CORRECTION

Figure $7(b)$ is now presented in its correct form.

A detailed erratum will appear at the end of the volume.

23 June 2008 